\title{
PGC-1 $\alpha$ promotes recovery after acute kidney injury during systemic inflammation in mice
}

\author{
Mei Tran, ${ }^{1}$ Denise Tam, ${ }^{1}$ Amit Bardia, ${ }^{1}$ Manoj Bhasin, ${ }^{2}$ Glenn C. Rowe, ${ }^{3}$ Ajay Kher, ${ }^{1}$ \\ Zsuzsanna K. Zsengeller, ${ }^{4}$ M. Reza Akhavan-Sharif, ${ }^{5}$ Eliyahu V. Khankin,, ${ }^{1}$ Magali Saintgeniez, ${ }^{6}$ \\ Sascha David, ${ }^{1}$ Deborah Burstein, ${ }^{5}$ S. Ananth Karumanchi, ${ }^{1,7,8}$ Isaac E. Stillman, ${ }^{4}$ \\ Zoltan Arany, ${ }^{3,8}$ and Samir M. Parikh ${ }^{1,8}$ \begin{abstract}
${ }^{5}$ Department of Radiology, Beth Israel Deaconess Medical Center, Boston, Massachusetts, USA. ${ }^{6}$ Schepens Eye Research Institute, Boston, Massachusetts, USA. ${ }^{7}$ Howard Hughes Medical Institute, Chevy Chase, Maryland, USA. ${ }^{8}$ Center for Vascular Biology Research, Beth Israel Deaconess Medical Center, Boston, Massachusetts, USA
\end{abstract} \\ 1Division of Nephrology, ${ }^{2}$ Division of Interdisciplinary Medicine and Biotechnology, ${ }^{3}$ The Cardiovascular Institute, 4Department of Pathology, and
}

\begin{abstract}
Sepsis-associated acute kidney injury (AKI) is a common and morbid condition that is distinguishable from typical ischemic renal injury by its paucity of tubular cell death. The mechanisms underlying renal dysfunction in individuals with sepsis-associated AKI are therefore less clear. Here we have shown that endotoxemia reduces oxygen delivery to the kidney, without changing tissue oxygen levels, suggesting reduced oxygen consumption by the kidney cells. Tubular mitochondria were swollen, and their function was impaired. Expression profiling showed that oxidative phosphorylation genes were selectively suppressed during sepsis-associated AKI and reactivated when global function was normalized. PPAR $\gamma$ coactivator $-1 \alpha(P G C-1 \alpha)$, a major regulator of mitochondrial biogenesis and metabolism, not only followed this pattern but was proportionally suppressed with the degree of renal impairment. Furthermore, tubular cells had reduced PGC-1 $\alpha$ expression and oxygen consumption in response to TNF- $\alpha$; however, excess PGC-1 $\alpha$ reversed the latter effect. Both global and tubule-specific PGC-1 $\alpha$-knockout mice had normal basal renal function but suffered persistent injury following endotoxemia. Our results demonstrate what we believe to be a novel mechanism for sepsis-associated AKI and suggest that PGC- $1 \alpha$ induction may be necessary for recovery from this disorder, identifying a potential new target for future therapeutic studies.
\end{abstract}

\section{Introduction}

Acute kidney injury (AKI) frequently complicates the care of hospitalized patients (1), and sepsis is among the 10 leading causes of mortality in adults in the United States (2). AKI affects approximately $50 \%$ of individuals with severe sepsis, and the combination of sepsis and AKI nearly doubles the risk of death from sepsis alone (3). No targeted therapies exist for sepsis-associated AKI, and recent large clinical trials have not identified an optimal form of supportive therapy for these patients $(4,5)$.

Acute reduction in renal function during severe sepsis arises from a combination of local ischemia, inflammation, and additional factors (6). Despite intense efforts to understand the unique features that distinguish the pathogenesis of sepsis-associated AKI from other forms of AKI, one important observation has not been fully addressed - i.e., both experimental models and human biopsy/necropsy series of septic AKI are characterized by a paucity of tubular cell death despite often severe impairment of global function (7-10).

The appreciation of the kidney as a highly metabolic organ can be traced back at least 50 years to the electron microscopy work of Rhodin and the biochemical balance studies of Krebs $(11,12)$. The energy required to move solutes against electrochemical gradients requires mitochondrial ATP generation, and AKI has broadly been described as a state of tubular ATP depletion (13). Not surprisingly,

Authorship note: Mei Tran, Denise Tam, and Amit Bardia contributed equally to this work.

Conflict of interest: The authors have declared that no conflict of interest exists. Citation for this article: J Clin Invest. 2011;121(10):4003-4014. doi:10.1172/JCI58662. the kidney is one of the major organs to express the mitochondrial biogenesis regulator PPAR $\gamma$ coactivator-1 $\alpha$ (PGC-1 $\alpha$ ) (14). Neither the role of mitochondrial dysregulation in sepsis-associated AKI nor that of PGC-1 $\alpha$ in normal or septic kidneys has been adequately investigated.

We hypothesized that organ dysfunction in sepsis-associated AKI could reflect a dynamic deficit in tubular mitochondrial function. To address this, we used 4 independent approaches - physiological measurements, ultrastructural analysis, assays of in situ electron transport enzymatic activity, and self-organizing maps (SOMs) of renal expression profiles - each of which confirmed the presence of marked mitochondrial defects following endotoxin challenge that resolve as renal function normalizes. Among genes that were suppressed during injury and restored during recovery was $P G C-1 \alpha$, but not its paralog, $P G C-1 \beta$. We therefore asked what role PGC- $1 \alpha$ played in tubular cell oxygen consumption and in the recovery from septic AKI using cultured tubule cells and global and tubule-specific knockout mice.

\section{Results}

Sustained intrarenal oxygenation in endotoxemic kidney. We administered $10 \mathrm{mg} / \mathrm{kg}$ LPS to 8-week-old adult male C57BL/6J mice. Eighteen hours after LPS treatment (Figure 1A), blood urea nitrogen (BUN, $32 \pm 3$ vs. $86 \pm 13 \mathrm{mg} / \mathrm{dl}, P<0.01)$ and serum creatinine $(\mathrm{Cr}, 0.19$ $\mathrm{mg} / \mathrm{dl} \pm 0.002$ vs. $0.26 \pm 0.03 \mathrm{mg} / \mathrm{dl}, P<0.05$ ) were significantly elevated. Transcript abundance of AKI biomarkers in whole kidney homogenates was consistent with intrinsic injury (Supplemental Figure 1A; supplemental material available online with this article; doi:10.1172/JCI58662DS1). Light microscopy showed minor changes 

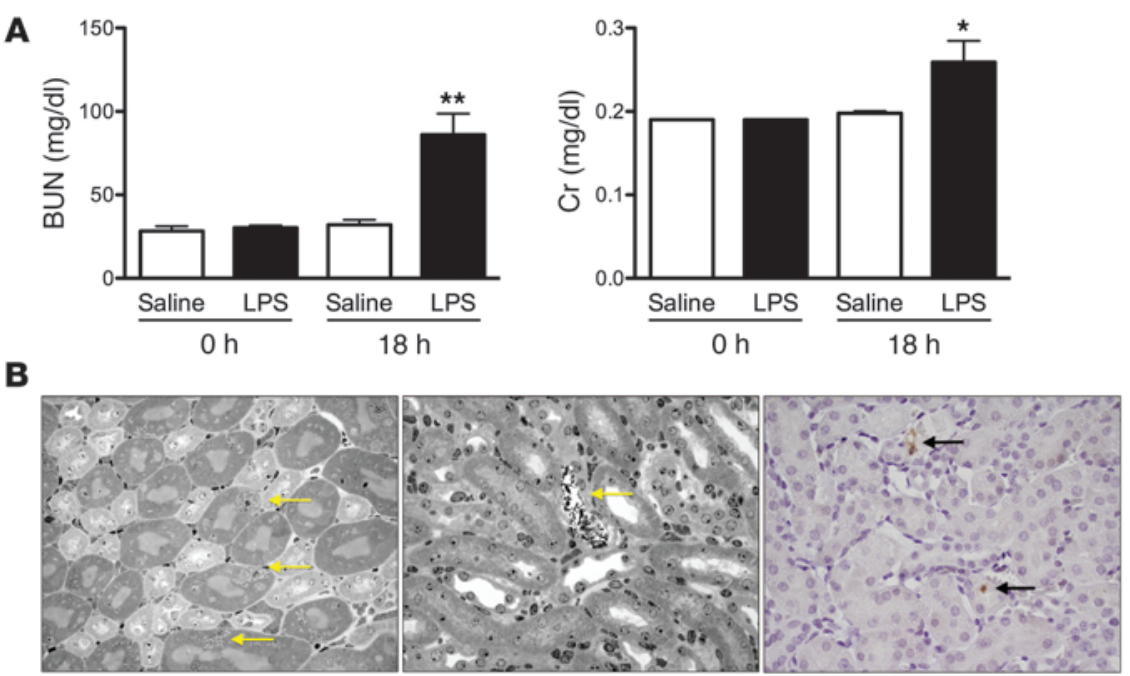

C
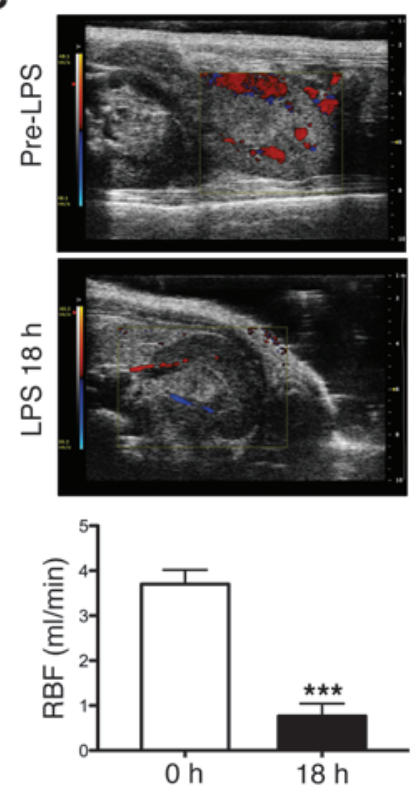

D
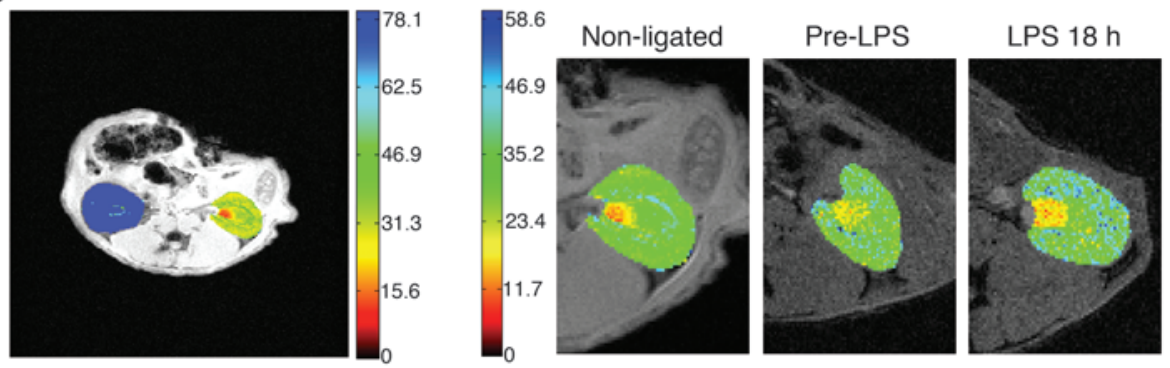

Figure 1

Characterization of the endotoxemia model. (A) $10 \mathrm{mg} / \mathrm{kg}$ of i.p. LPS triggers a rise in BUN and serum creatinine $(\mathrm{Cr})$ at $18 \mathrm{hours} ; n=4-11$ per group. (B) Histological signs of injury in the LPS model are scarce. Left: Yellow arrows indicate individual proximal tubule cells with vacuolar changes; middle: yellow arrow points to a single necrotic cortical tubule; right: immunohistochemistry for cleaved caspase-3 (black arrows indicate positive cells) identifies scant apoptosis (representative images of $n=6$ mice). Original magnification, $\times 40$. (C) Color Doppler images (red indicates arterial flow; blue, venous flow) and renal artery blood flow (RBF) in $n=5$ mice before and 18 hours after LPS administration. (D) BOLD MRI images for serial measurement of intrarenal oxygenation. Upper left: Axial R2* map of mouse with partial ligation of the left kidney and non-ligated contralateral kidney (R2* scale clipped at $80 \mathrm{~Hz}$ ); upper right: axial R2* maps of non-ligated, pre-LPS, and 18 hours post-LPS kidney showing no major changes in overall or regional R2* values (R2* scale $0-60 \mathrm{~Hz}$ ); bottom panel: overall R2* values for $n=5$ mice before and 18 hours after LPS treatment showing no significant difference. ${ }^{*} P<0.05,{ }^{* *} P<0.01,{ }^{* \star *} P<0.001$ versus control or pre-LPS condition.

- micro-vacuolization in less than $10 \%$ of proximal tubular cells, minimal focal necrosis, and no apoptosis (Figure 1B). In fact, the vast majority of tubular cells appeared histologically normal.

We measured renal perfusion by micro-ultrasound performed before and 18 hours after LPS administration. Renal artery blood flow declined markedly in LPS-treated mice (Figure 1C). Since blood flow is the major determinant of oxygen delivery, we anticipated renal hypoxia in the endotoxemic mice. To test this, we applied blood oxygen level-dependent (BOLD) MRI, a non-invasive technique that utilizes the difference in magnetization between oxyhemoglobin (diamagnetic) and deoxyhemoglobin (paramagnetic) to calculate a parameter, $\mathrm{R} 2$ *, that is directly proportional to the tissue content of deoxyhemoglobin, which in turn reflects blood and tissue $\mathrm{PO}_{2}(15)$. We found that pre-LPS R2* values were indistinguishable from R2* values 18 hours after LPS $(36 \pm 3.9$ vs. $36.4 \pm 4.2 \mathrm{~Hz}, P=0.91$ ) (Figure $1 \mathrm{D}$ ), whereas a positive control, unilateral renal artery ligation, showed marked elevation of R2*. We further confirmed the maintenance of tubular oxygenation by 
A
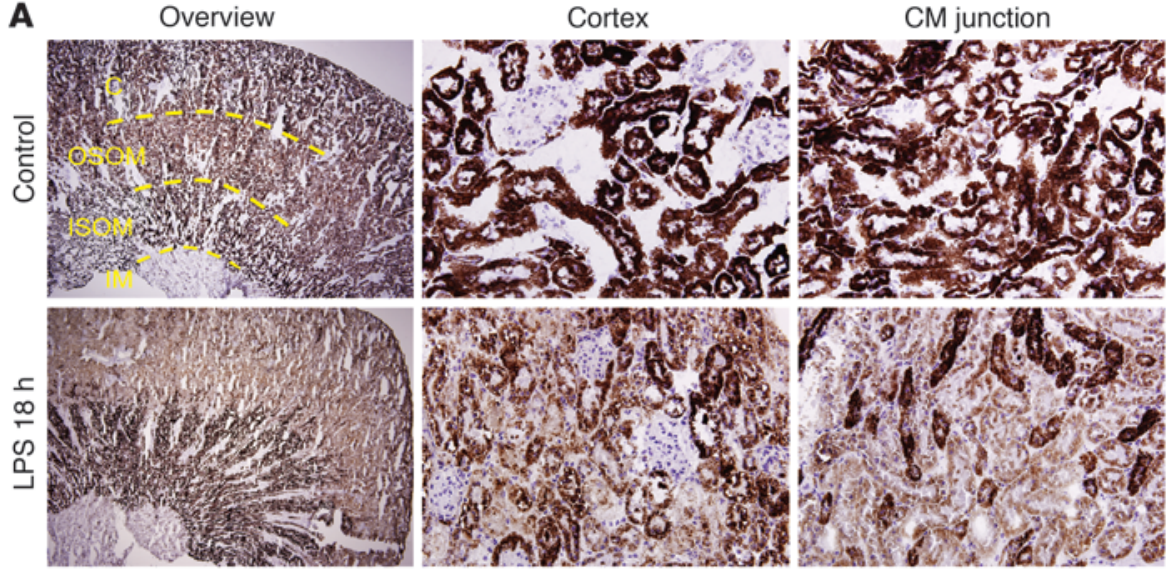

B

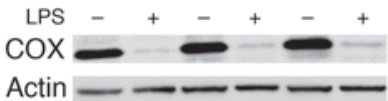

C
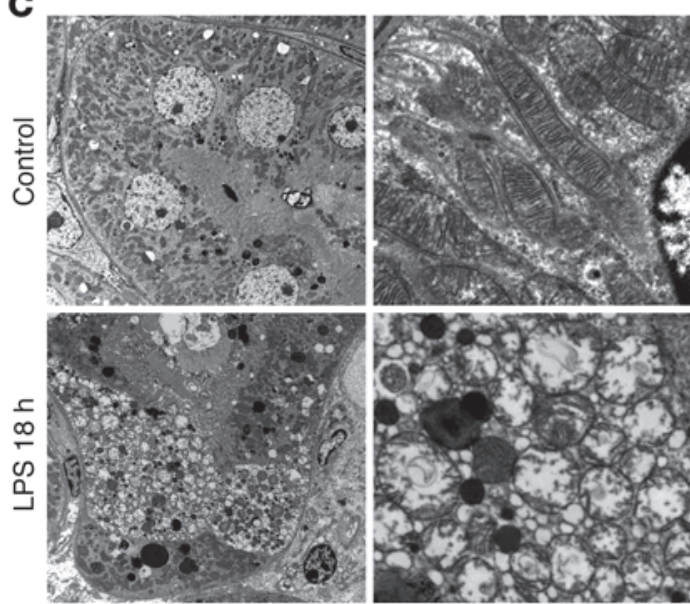

\section{Figure 2}

Regional distribution and severity of mitochondrial dysfunction and injury. (A) Staining (brown) for cytochrome $c$ oxidase enzyme activity on slices from snap-frozen kidneys 18 hours after vehicle or LPS treatment. Cortex (C) and inner stripe of the outer medulla (ISOM) have the most intense staining, followed by outer stripe of the outer medulla (OSOM), with the weakest activity in the inner medulla (IM). Enzymatic activity is greatly reduced following LPS. Original magnification, $\times 4$ (overview); $\times 40$ (cortex, corticomedullary [CM] junction). (B) Western blot on whole kidney lysates for cytochrome $C$ oxidase. (C) Transmission EM of proximal tubules demonstrating swelling of mitochondria and rarefaction of cristae. $n=3-6$ mice per condition. Original magnification, $\times 3,000$ (left); ×20,000 (right). measuring the abundance of HIF-1 $\alpha$, the hypoxia-inducible factor isoform previously shown to be specific for tubular hypoxia in vivo (16), in kidney lysates (Supplemental Figure 1B). The absence of HIF-1 $\alpha$ elevation accumulation 18 hours after LPS treatment suggested that renal hypoxia, if present, was not substantial enough to induce its measurable accumulation. These experiments show that endotoxemic renal injury is not associated with widespread tubular cell death and that renal tissue oxygenation is not overtly reduced despite less oxygen delivery.

Biochemical and structural evidence of proximal tubule mitochondrial dysfunction. Based on the mismatch between oxygen delivery and tissue oxygenation, we hypothesized that reduced renal oxygen consumption in endotoxemia could reflect mitochondrial dysfunction. To assess this, we measured the in situ activity of the electron transport chain enzyme complex cytochrome $c$ oxidase, with and without LPS challenge (Figure 2A). In this assay, incubation of tissue slices with acid and cytochrome $c$ provides substrates for the enzyme complex, whose activity is then detectable as brown staining. The degree of brown staining therefore reflects cytochrome $c$ oxidase activity, which was found to be high in cortical tubules and dramatically reduced 18 hours after LPS. The regions most prominently affected were the cortex and outer stripe of the outer medulla, whereas the inner stripe of the outer medulla was less affected by LPS. The reduced activity of cytochrome $c$ oxidase was associated with decreased protein abundance (Figure $2 \mathrm{~B}$ ). In the surgical model of sepsis, cecal ligation and perforation (CLP), we observed vacuolization of proximal tubular cells with little cell death, as previously reported $(6,10)$ and analogous to the renal pathology following LPS (Figure 1B). Moreover, we saw remarkably similar changes in cytochrome $c$ oxidase activity within the kidney following CLP, with the most prominent reduction in enzyme activity again affecting the cortex and outer stripe (Supplemental Figure 2A).

Ultrastructural studies of LPS-challenged kidneys revealed individual proximal tubular cells with swollen mitochondria and rarefied cristae (Figure 2C). Finally, we also performed an in situ study of NADH dehydrogenase localization (Supplemental Figure 2B), which mirrored the LPS-induced reduction in cytochrome $c$ oxidase activity. Together, these results provide strong evidence of sepsis-induced perturbations in the function and structure of renal tubular mitochondria.

Expression profiling analyses of endotoxemic kidneys. Resuscitation of LPS-treated mice with $10 \mathrm{ml} / \mathrm{kg}$ warmed sterile $0.9 \%$ saline at the 18-hour mark enhanced the survival of our mice to greater than $80 \%$, enabling us to study outcomes of endotoxemic AKI. 
A
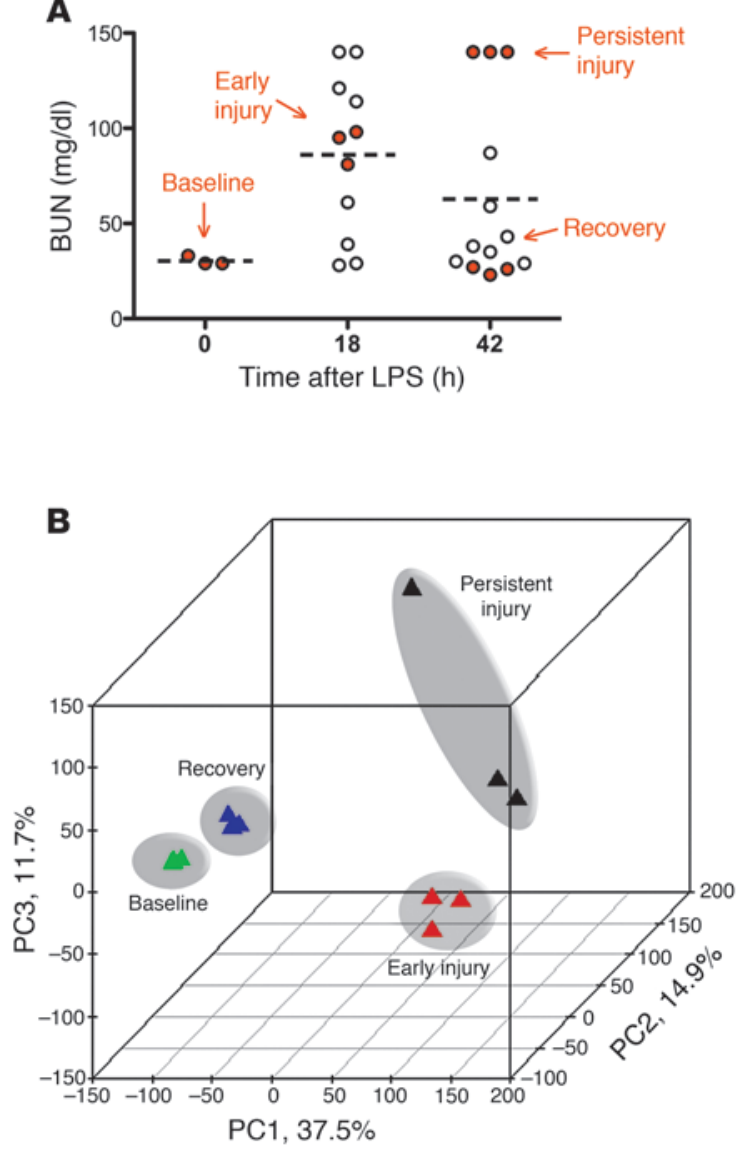

C

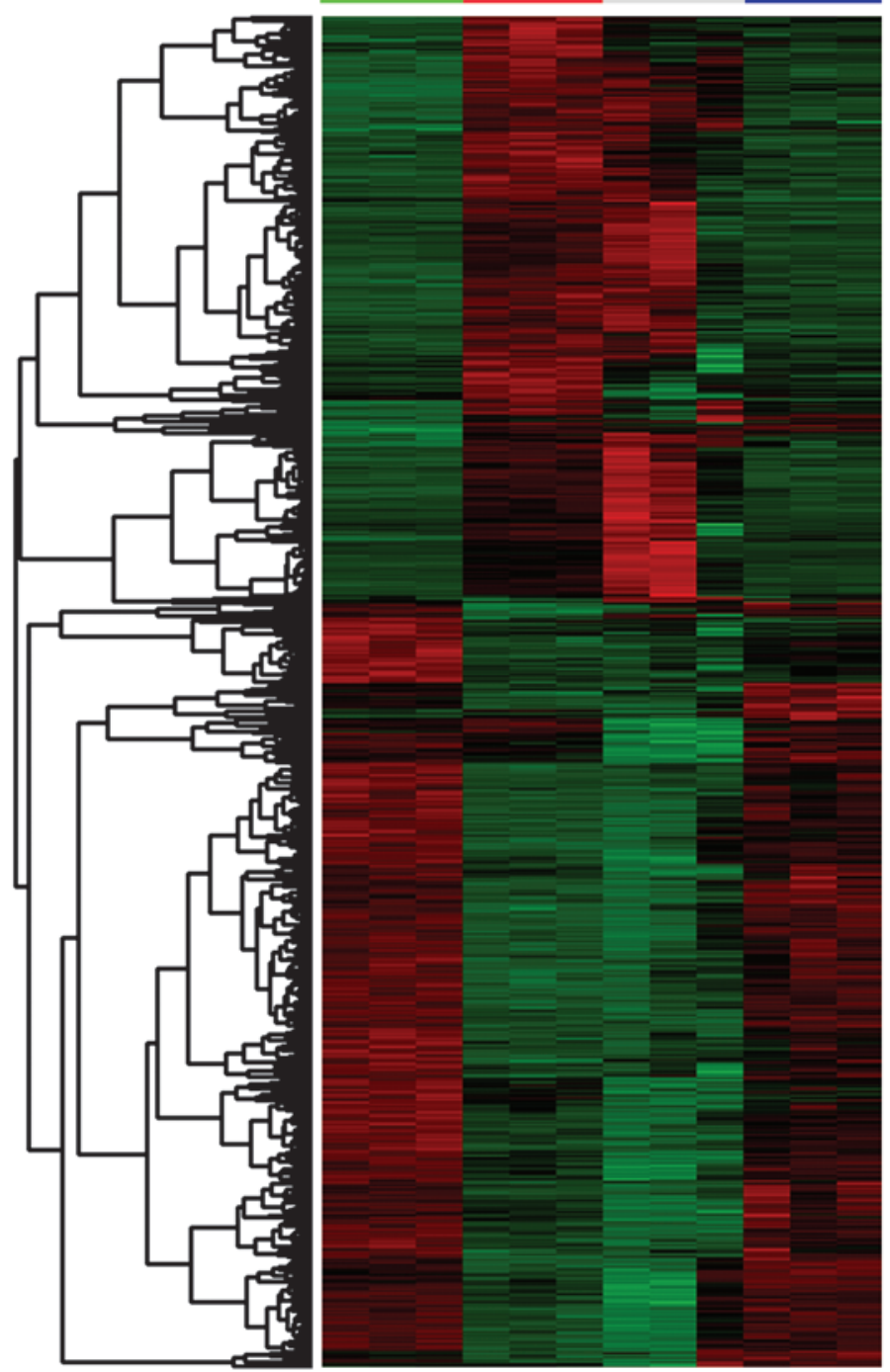

\section{Figure 3}

Similarities in expression profiles between early and persistent injury and between baseline and recovery states. (A) Eighteen hours after LPS, mice received $10 \mathrm{ml} / \mathrm{kg}$ saline, and by 42 hours after LPS, the majority had recovered from AKI. Kidneys from $n=3$ animals per indicated condition (baseline, early injury, persistent injury, recovery; red circles) were studied by expression profiling. Dashed horizontal lines indicate mean values. (B) PCA of the transcriptional data from each condition. The percentage values indicate the proportion of total variance described by the corresponding principal component. (C) Genes were selected using supervised analysis on the basis of 1-way ANOVA of the 4 conditions - baseline, early injury, persistent injury, and recovery. Columns represent the samples, and rows represent the genes, with expression demonstrated by pseudocolor scale $(-3=$ green, $+3=$ red $)$.

The majority of mice recovered normal function by 42 hours after LPS, but some had persistent AKI. To study this heterogeneity, we performed expression profiling on kidney-derived RNA obtained at time 0 (baseline), 18 hours after LPS (early injury), and in two groups 42 hours after LPS (persistent injury and recovery, Figure $3 \mathrm{~A})$. In order to determine the relationships among these 4 different experimental conditions and the 3 replicates within each condition, we performed an unsupervised analysis of the microarray data. By principal component analysis (PCA) (Figure 3B), we were able to demonstrate that samples segregated on the basis of injury status along primary component 1 (PC1), which accounted for
$37.5 \%$ of the variation between samples. PC2 and PC 3 accounted for $14.9 \%$ and $11.7 \%$ of the total variance across all 12 samples. We identified 7,202 significantly differentially expressed genes by employing 1 -way ANOVA on the 4 conditions $(P<0.001)$. To reduce the likelihood of false positives, this list was filtered to remove genes with a standard deviation less than 5 across groups (Figure 3C), yielding 5,186 unique differentially expressed transcripts corresponding to 3,910 RefSeq gene symbols.

As opposed to the standard binary comparison of arrays between two experimental conditions, we were interested in genes that simultaneously fulfilled several expression criteria, for example: (a) elevated 

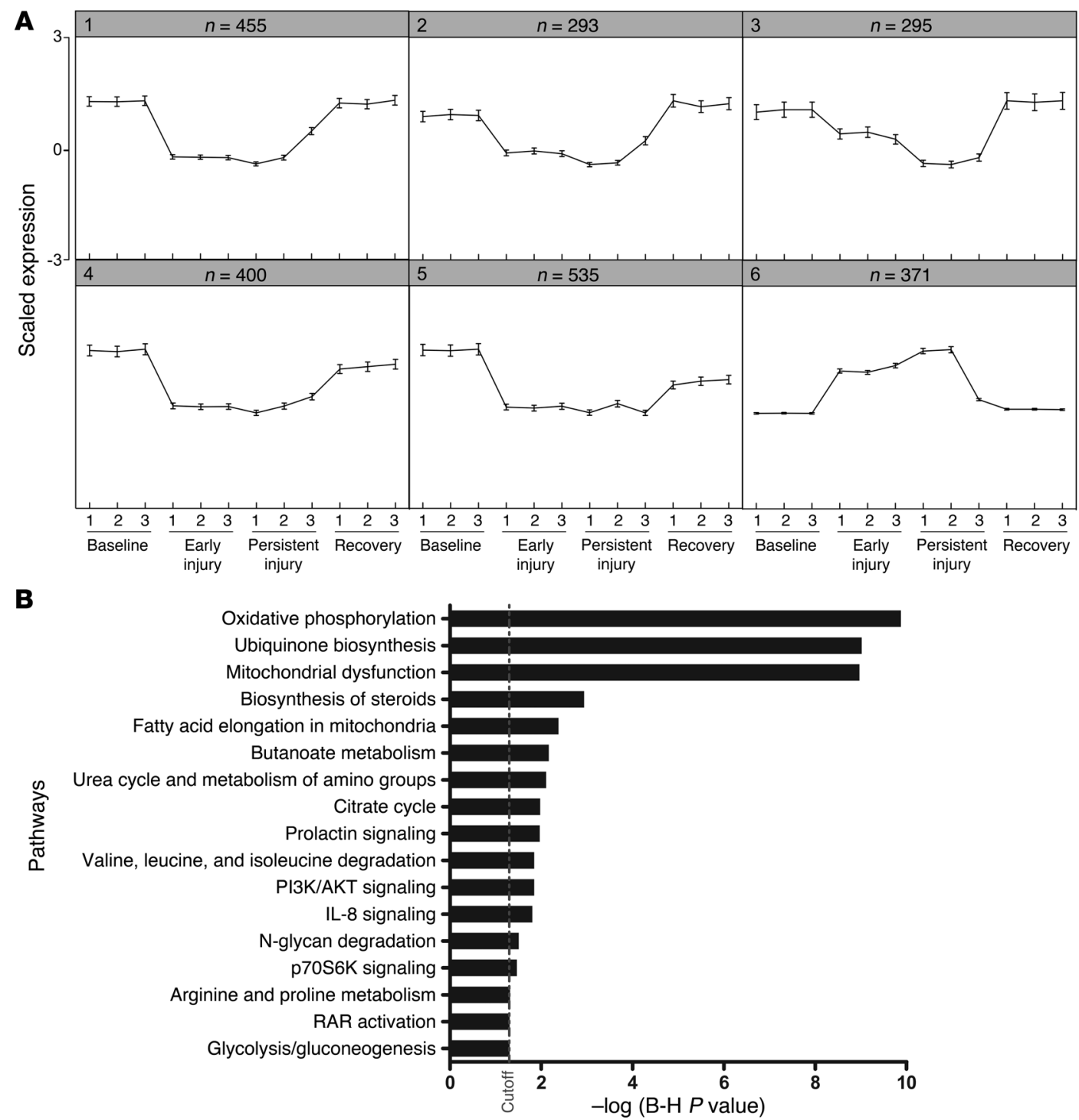

\section{Figure 4}

SOM and pathway analyses. (A) Array results were analyzed by a SOM algorithm for 50 target groups. Shown are the 6 strongest clusters of genes with similar relative expression profiles across conditions. Number of transcripts per cluster is indicated above each graph. (B) Analysis of pathways enriched in SOM clusters identified oxidative phosphorylation as the most strongly over-represented biological group. Each bar represents a significantly enriched pathway as determined using the Benjamini-Hochberg hypothesis (B-H) corrected $P$ value (shown on $x$ axis).

in early injury, (b) elevated in persistent injury, and (c) reduced to baseline levels in recovery. To do this, we used SOMs to detect groups of differentially expressed genes with similar expression patterns across the 4 conditions, arbitrarily drawing 50 separate maps according to Pearson correlation coefficient-based distance metrics. Based on significant similarity in the majority of the expression patterns, we further reduced the number of distinct expression profiles to 6 clusters (Figure 4A). Most clusters were suppressed in early and persistent injury and restored to normal levels during recovery; one cluster, no. 6, varied oppositely, representing a group of genes whose expression was elevated during early AKI and persistent AKI and returned to normal levels during recovery (Supplemental Table 1).

To identify biological patterns in the SOM clusters, we performed canonical pathway enrichment using Ingenuity Pathway
Analysis (IPA) tools and calculated a multiple-test adjusted $P$ value for each pathway according to the fit of our data to the IPA database. Significant enrichment was observed in several signaling, biosynthetic, and metabolic pathways (Figure 4B). The most overrepresented biological group among these clusters was oxidative phosphorylation (corrected $P=1.3 \times 10^{-10}$ ).

Suppression of mitochondrial gene transcripts is proportional to the degree of renal dysfunction. Based on the confluence of physiological, structural, biochemical, and molecular findings suggesting a role for mitochondrial dysfunction in the experimentally septic kidney, we measured the intrarenal expression of mitochondrial genes by quantitative PCR and compared transcript abundance with the degree of functional impairment. The mitochondrial biogenesis regulator $P G C-1 \alpha$, but not its paralog $P G C-1 \beta$, was suppressed proportionally 

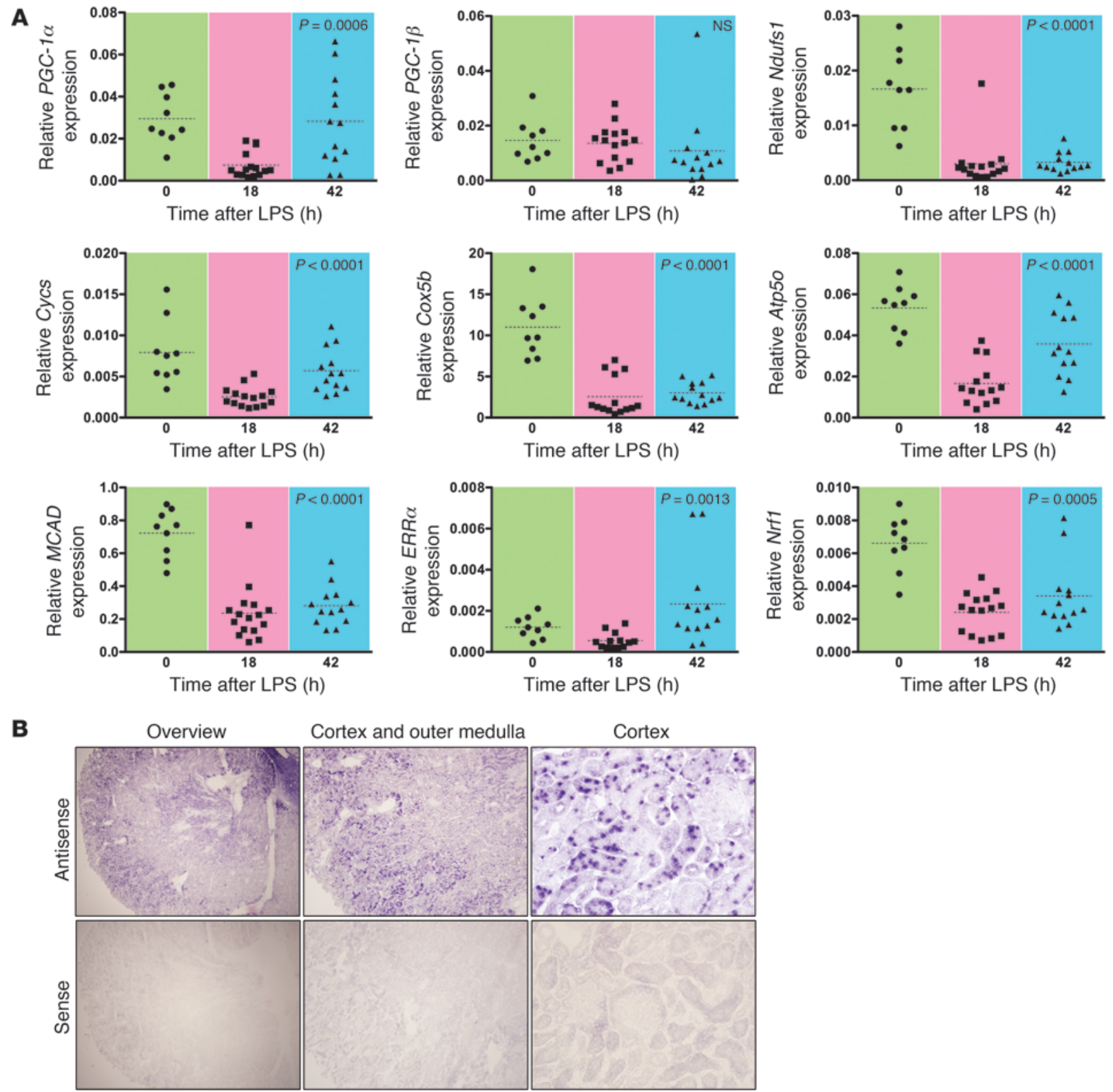

\section{Figure 5}

Coordinated changes in the expression of $P G C-1 \alpha$ and downstream genes. (A) Expression level for $P G C$ - $1 \alpha$ and other genes related to mitochondrial biogenesis and function was measured in RNA prepared from kidneys harvested at the indicated time points after LPS (reported as abundance relative to $\beta$-actin). $P$ values were determined by ANOVA. Note that $P G C-1 \beta$ does not significantly vary over time, unlike $P G C$-1 $\alpha$. $n=35-37$ data points per gene studied. MCAD, medium-chain acyl-CoA dehydrogenase; $E R R \alpha$, estrogen related receptor $\alpha$; Nrf1, nuclear respiratory factor 1. (B) In situ hybridization for PGC-1 $\alpha$ (antisense) or sense probe performed on snap-frozen kidney sections. Representative images shown (overview; original magnification, $\times 4$ ) with higher-power image of the cortex and outer medulla (original magnification, $\times 10$ ) and high-power image of the cortex (original magnification, $\times 40$ ), where the absence of glomerular staining and strong positivity of proximal tubules are evident.

to the degree of functional impairment following LPS challenge (Supplemental Figure 3A; $P G C-1 \alpha: r=-0.5, P=0.0016$ vs. $P G C-1 \beta$ : $r=-0.32, P=\mathrm{NS})$. Similarly, transcripts encoding electron transport chain components, fatty acid oxidation enzymes, and metabolic transcription factors were all significantly and proportionally suppressed. The correlation between severity of renal dysfunction and renal $P G C-1 \alpha$ expression was also confirmed following the CLP model (Supplemental Figure 3B; $r=-0.9286, P=0.0067$ ).
When analyzed temporally (Figure 5A) the expression of $P G C-1 \alpha$ $(P=0.0006)$, but not $P G C-1 \beta(P=\mathrm{NS})$, rebounded to normal levels by 42 hours after LPS. Among electron transport chain components, the expression of cytochrome $c$, somatic (Cycs; $P<0.0001)$ and ATP synthase subunit $\mathrm{O}$, mitochondrial (Atp5o; $P<0.0001)$ showed the most recovery by the latest time point (Figure 5A). Correlation among the expression of $P G C-1 \alpha$ and its downstream electron chain component genes was also 


\section{Table 1}

Covariation of PGC-1a expression and expression of downstream genes

\begin{tabular}{lccccc} 
& PGC-1 $\alpha$ & Ndufs1 & Cycs & Atp5o & Cox5b \\
MCAD & $0.41^{\mathrm{A}}$ & $0.79^{\mathrm{B}}$ & $0.55^{\mathrm{C}}$ & $0.73^{\mathrm{B}}$ & $0.76^{\mathrm{B}}$ \\
Cox5b & $0.31^{\mathrm{D}}$ & $0.66^{\mathrm{B}}$ & $0.71^{\mathrm{B}}$ & $0.71^{\mathrm{B}}$ & \\
Atp5o & $0.61^{\mathrm{B}}$ & $0.66^{\mathrm{B}}$ & $0.66^{\mathrm{B}}$ & & \\
Cycs & $0.57^{\mathrm{C}}$ & $0.35^{\mathrm{A}}$ & & & \\
Ndufs1 & $0.46^{\mathrm{E}}$ & & & & \\
\hline
\end{tabular}

Matrix of $P G C-1 \alpha$ and downstream gene expression where numbers indicate Spearman correlation coefficient. ${ }^{A} P<0.05$. ${ }^{B} P<0.0001$.

$\mathrm{C} P<0.01$. DNS. $\mathrm{E} P<0.001$.

strong and highly significant, suggesting a coordinated metabolic response (Table 1 ).

Given the role of $P G C-1 \alpha$ as an orchestrator of mitochondrial biogenesis and metabolism, we performed in situ hybridization for $P G C-1 \alpha$ (Figure 5B). The highest expression was observed in the proximal tubule, and cortical tubules had higher expression than medullary tubules. In the medulla, the thick ascending limb had the strongest signal. As expected, the pattern of PGC-1 $\alpha$ expression overlapped with the intrarenal distribution of oxidative phosphorylation activity (Figure 2A). We also confirmed the endotoxemic suppression of PGC-1 $\alpha$ protein by Western blot analysis (Supplemental Figure 4) and performed a time course experiment out to 42 hours in LPS-treated mice that received saline resuscitation $(10 \mathrm{ml} / \mathrm{kg})$ at 18 hours after LPS treatment to document the temporal profile of changes in renal function and PGC-1 $\alpha$ (Supplemental Figure 5).

Prevention of cytokine-induced tubular respiratory suppression by excess PGC-1 $\alpha$. To test whether a direct relationship exists between acute inflammation and reduced tubular oxygen consumption, we studied primary human renal proximal tubular epithelial cells (RPTECs). When cells were treated with TNF- $\alpha, P G C-1 \alpha$ and its target genes were markedly suppressed (Figure 6A), consistent with experiments we performed in a transformed proximal tubule cell line (Supplemental Figure 6) and previous studies by others (17-19). Next, we studied the oxygen consumption of TNF- $\alpha-$ treated RPTECs (Figure 6B). Both basal oxygen consumption and uncoupled respiration were suppressed in a dose- and time-dependent fashion (Figure 6C). We then applied viral delivery of murine $P G C-1 \alpha$, a manipulation previously shown to increase the abundance of morphologically and functionally normal mitochondria (20). Induction of $P G C-1 \alpha$ by adenovirus restored both the level of oxygen consumption (Figure 6D) and the expression of downstream target genes (Figure 6E) to those of untreated RPTECs.

Effects of global and proximal tubule-specific genetic depletion of PGC- $1 \alpha$. Having found a strong negative correlation between BUN and $P G C-1 \alpha$ expression in septic AKI (Supplemental Figure 3, A and $\mathrm{B}$ ) and a recovery of its expression as AKI resolves (Figure 5A), we hypothesized that the suppression of $P G C-1 \alpha$ may be retarding recovery of renal function. To test this, we first used global PGC-1 $\alpha-$ knockout mice (21), for which the renal phenotype has not yet been described (22). At baseline, 8-week-old null mice had no major detectable ultrastructural defect in mitochondria or change in renal function compared with their wild-type littermates (Supplemental Figure 7A). To study its role in recovery from AKI, we again administered $10 \mathrm{ml} / \mathrm{kg}$ saline at 18 hours after LPS. Null mice had impaired renal function at 42 hours after LPS compared with their wild-type littermates (BUN $81.7 \pm 14.4$ vs. $47.9 \pm 7.7 \mathrm{mg} / \mathrm{dl}$, $P<0.05$, Figure 7A). To rule out an effect of extrarenal interaction of $P G C-1 \alpha$ depletion with endotoxin exposure, we developed a proximal tubule-specific knockout model (Figure 7B) by breeding PGC-1 $\alpha$ floxed mice to Sglt2-Cre mice. Tubule-specific knockout mice had no major baseline functional renal deficit at 8 weeks of age (Supplemental Figure 7B) but, when exposed to the stress of endotoxemia, developed persistent AKI compared with nonknockouts (BUN $75.7 \pm 7.3$ vs. $40.5 \pm 2.3 \mathrm{mg} / \mathrm{dl}, P<0.01$, Figure $7 \mathrm{C})$. Together, these results provide strong evidence that endotoxemic suppression of $P G C-1 \alpha$ is a critical event that influences the duration of functional impairment.

\section{Discussion}

In the present work, complementary physiological, biochemical, ultrastructural, and molecular profiling studies strongly and independently suggest that mitochondrial dysfunction is a major contributor to the renal functional impairment that occurs in sepsis. Moreover, PGC- $1 \alpha$ appears to be an important regulator in this disorder, becoming suppressed in proportion to the degree of organ dysfunction and rising back to pre-injury levels as function normalizes. The importance of PGC- $1 \alpha$ 's dynamic regulation during the course of endotoxemia is evidenced by the persistence of AKI in global and proximal tubule-specific knockouts.

The pathogenesis of AKI in sepsis is multifactorial, with contributions from renal hypoperfusion $(23,24)$, parenchymal responses to circulating cytokines (25-27), microvascular injury and inflammation, and microthrombi (28). However, none of these mechanisms offers a complete explanation for why the degree of renal dysfunction in sepsis is disproportionate to the amount of cell death that has generally been observed. Our data show that light microscopy evaluation alone inadequately reflects the molecular disruptions that characterize the endotoxemic kidney. Whereas vacuolization was apparent only in less than $10 \%$ of proximal tubular cells, cytochrome $c$ oxidase activity (Figure 2 and Supplemental Figure 2) and $P G C-1 \alpha$ expression (Figure 5) were more broadly suppressed. Since our results suggest that the "vacuoles" are actually swollen mitochondria, we infer that this visible change is an extreme manifestation of widespread mitochondrial dysfunction that is more sensitively measured by molecular methods. In other words, only a small number of cells may progress so far into mitochondrial dysfunction as to display structural changes to the mitochondria. Analogously, in toxic or ischemic forms of AKI, the observed cell death may only be the "tip of the iceberg," representing the culmination of more widespread subcellular molecular changes.

Although mitochondrial dysfunction and sustained local oxygenation have been described in tissues obtained from experimentally septic mammals (heart, skeletal muscle, and liver) and human subjects with sepsis (skeletal muscle biopsies) (29-34), less work has been done to test this hypothesis in sepsis-associated AKI. Prior studies have shown that in the first $1-4$ hours after intravenous endotoxin exposure, intrarenal oxygen consumption is unchanged despite reduced delivery (35-37). Our results at a later time may reflect a transition to mitochondrial dysfunction as endotoxemia progresses. Since electron transport enzymes are rapidly inhibited by the abundant reactive oxygen (ROS) and nitrogen species generated in sepsis, it is likely that the mitochondrial dysfunction occurs post-transcriptionally, i.e., independent of PGC-1 $\alpha$ 's suppression. Damaged mitochondria, in turn, can become a source of increased oxidative stress to cells, accelerating the cycle of free radical injury. 


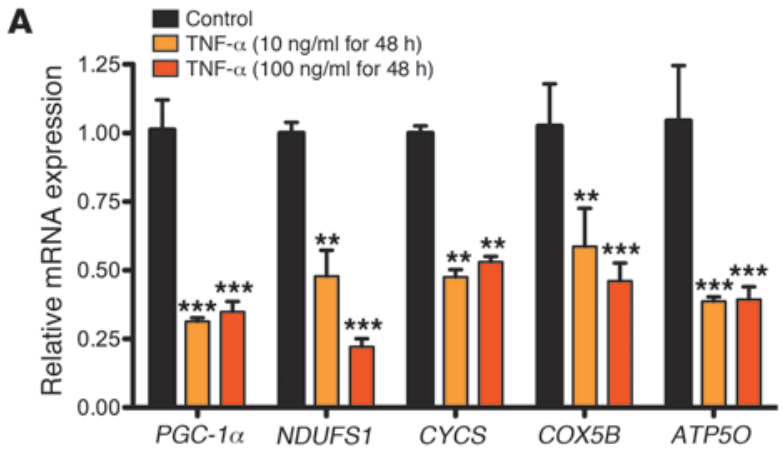

B

C
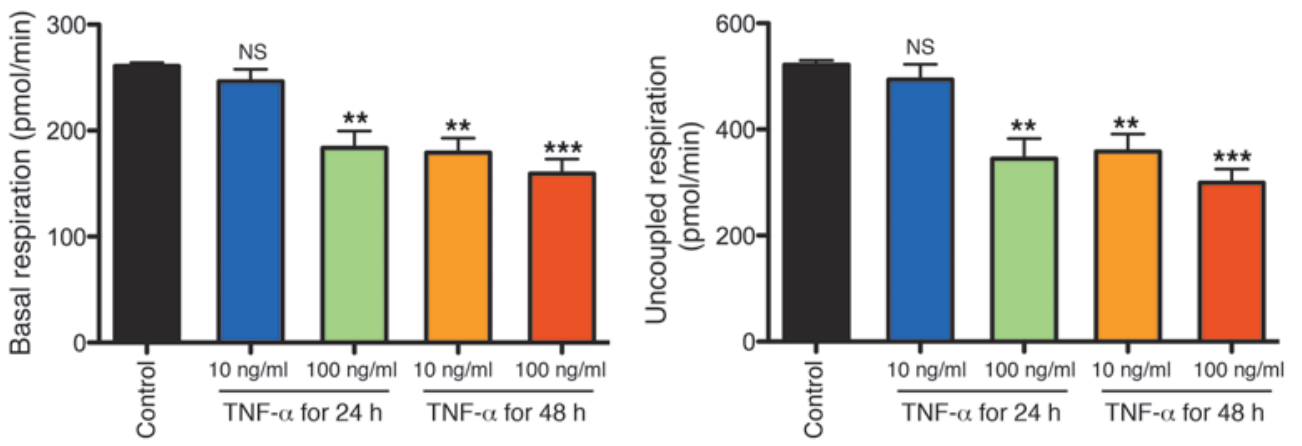

D
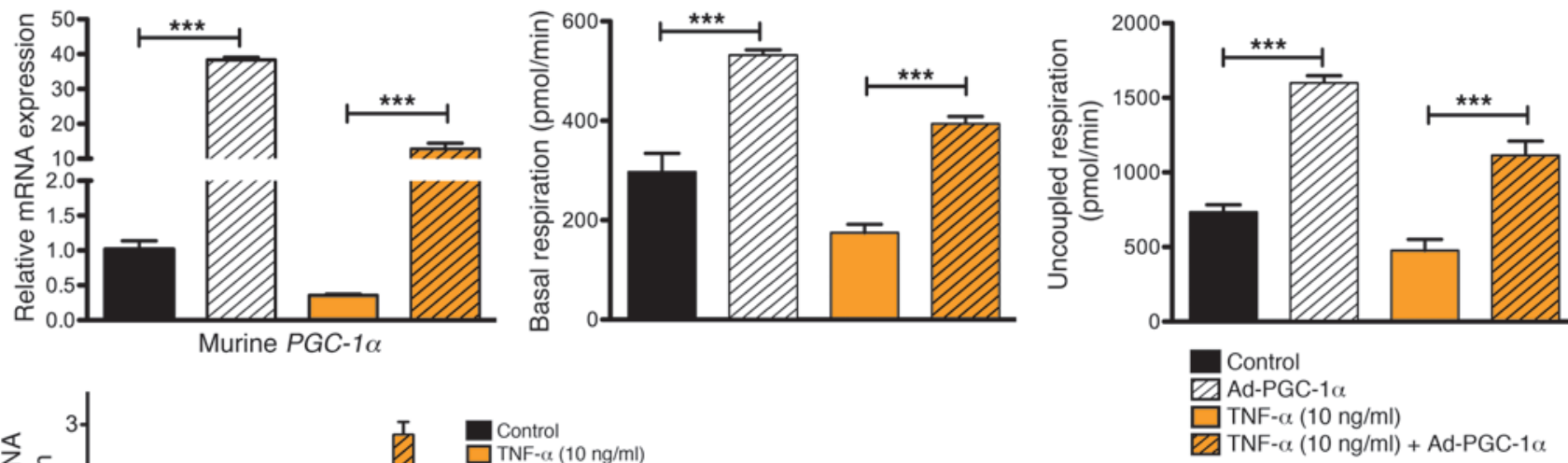

E
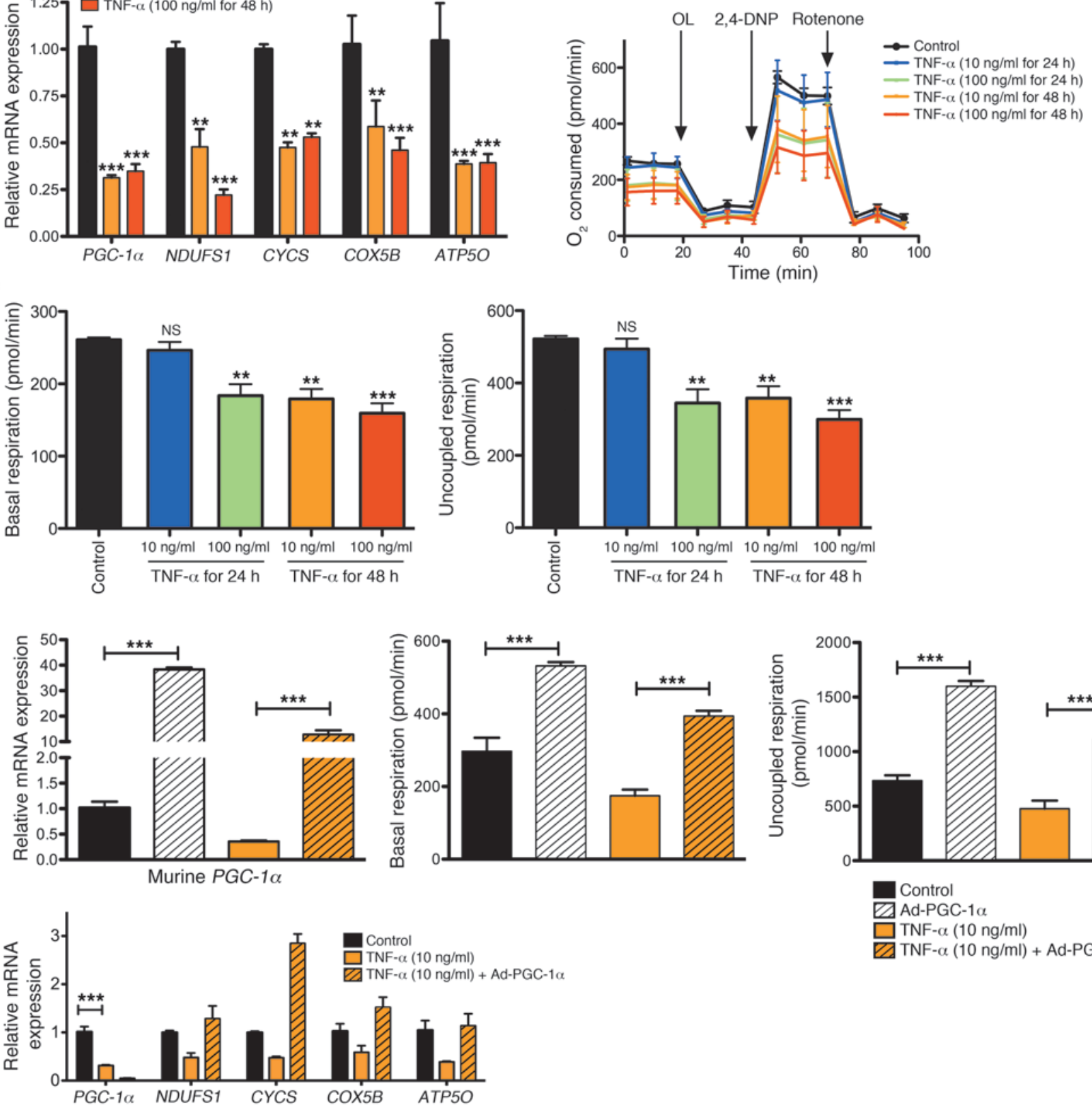

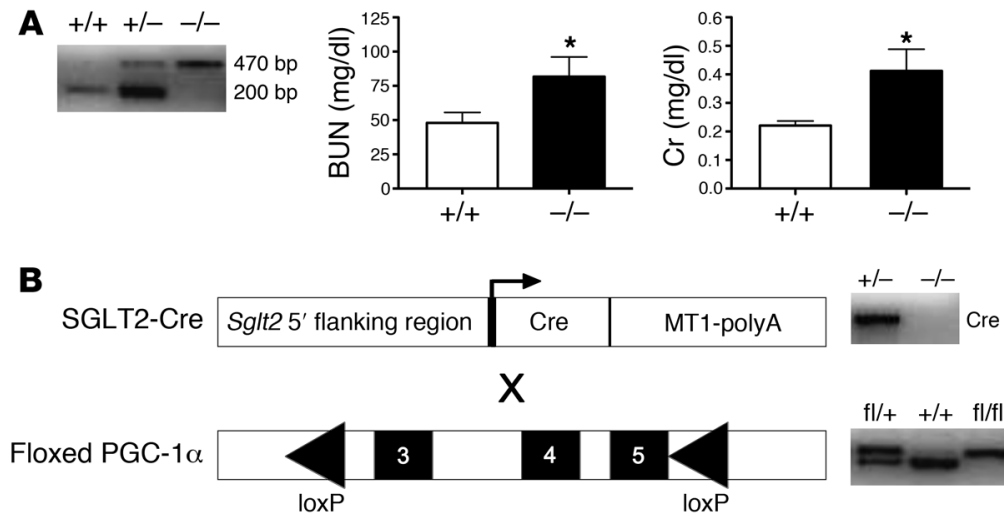

$\mathrm{fl} /+++\mathrm{fl} / \mathrm{fl}$
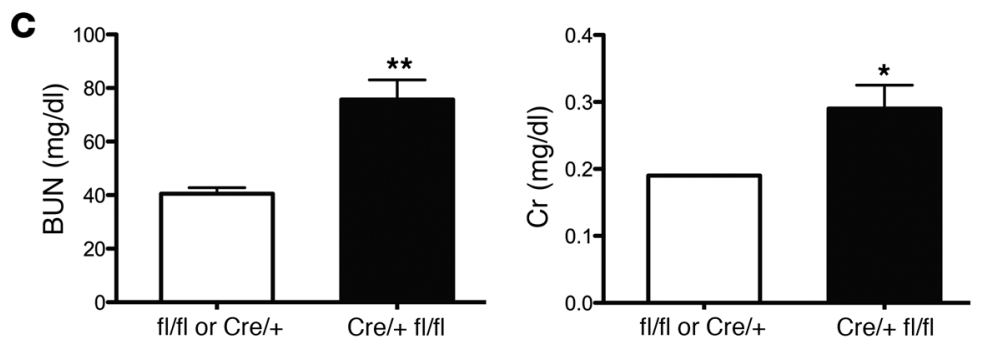

\section{Figure 7}

Persistent endotoxemic AKI in global and proximal tubule-specific $P G C-1 \alpha$-knockout mice. (A) Eightto 10-week-old male global knockouts for $P G C-1 \alpha$ $(-/-)$ or their wild-type littermates $(+/+)$ were given 10 $\mathrm{mg} / \mathrm{kg}$ LPS, then $10 \mathrm{ml} / \mathrm{kg}$ saline 18 hours after LPS. Renal function was measured 42 hours after LPS (i.e., 24 hours after saline resuscitation). BUN and $\mathrm{Cr}$ were significantly elevated in knockouts versus wild-type littermates. (B) Schematic for generating proximal tubule-specific knockouts of $P G C-1 \alpha$. Mice expressing Cre recombinase under the control of the Sglt2 promoter (Sglt2-Cre) were crossed with mice bearing loxP sites surrounding exons $3-5$ of $P G C-1 \alpha$ (floxed $P G C-1 \alpha$ ), and progeny were genotyped as shown. (C) Eight- to 10-week-old male Sglt2Cre/+ $P G C-1 \alpha^{f / / f l}(\mathrm{Cre} /+\mathrm{fl} / \mathrm{fl})$ mice experienced persistent $\mathrm{AKI}$ in the saline-resuscitated model compared with age- and sex-matched $\mathrm{Cre} /+$ or $\mathrm{fl} / \mathrm{fl}$ controls. $n \geq 14$ per group. ${ }^{\star} P<0.05,{ }^{\star \star} P<0.01$.
$P G C-1 \alpha$ is also sufficient to counteract the suppression of target genes in vitro (Figure 6). But other signals that interact with the mitochondrial biogenesis pathway are likely also present in the milieu of in vivo sepsis $(17,38)$. This may help explain why the PGC-1 $\alpha$-dependent genes cytochrome $c$ oxidase, subunit 5B $(C O X 5 B)$ and NADH-ubiquinone oxidoreductase $75 \mathrm{kDa}$ subunit, mitochondrial (NDUFS1) were restored by ectopic expression of $P G C-1 \alpha$ in cell culture, but did not return to normal levels in vivo even as $P G C-1 \alpha$ itself had rebounded.

The regulation of PGC- $1 \alpha$ in sepsis may include not only the addition of suppressive factors such as LPS and TNF- $\alpha$, but also the withdrawal of baseline inducers that have been identified in non-renal model systems - e.g., $\beta$-adrenergic signaling, AMP kinase activation, and sirtuins (39). Restoration of PGC-1 $\alpha$ 's expression may proceed through reversal of the same mechanisms, although this remains to be formally demonstrated. The major salutary effect of restored PGC- $1 \alpha$ expression is likely to be the biogenesis of new, healthy, coupled mitochondria, but other downstream effects, such as the induction of endogenous antioxidant defenses (40), may also be important. Given the complexity of PGC- $1 \alpha$ 's regulation and the assortment of molecular pathways that are deranged in sepsis, identifying a single signaling mechanism for sepsis-mediated PGC- $1 \alpha$ regulation may be difficult or even an oversimplification.

Basal expression of PGC- $1 \alpha$ appears to be higher in the proximal tubule compared with other metabolic tissues, including brown fat and cardiac muscle (our unpublished observations), suggesting a unique combination of local factors favoring tonic induction of the gene. While those factors have yet to be identified, independent lines of evidence also support the notion that active maintenance of healthy mitochondria is critically important to the preservation of normal renal function: (a) chronic tubulopathy is highly prevalent in inherited mitochondrial disorders (41); (b) induction of a PGC-1 $\alpha$ target, PPAR $\alpha$, prevents ischemic or toxic renal injury (42); (c) antagonism of a mitochondrial fragmentation pathway blunts ischemic or toxic renal injury (43); and (d) heterologously expressed PGC-1 $\alpha$ promotes mitochondrial recovery after exposure to oxidants in vitro (44). Together, these studies suggest that sustained activation of mitochondrial biogenesis may constantly replenish the intracellular pool of mitochondria and thereby prevent or ameliorate tubular injury from diverse sources such as ischemia/reperfusion, toxins, and oxidants.

Future studies are merited. First, further work should illuminate additional factors in sepsis that regulate PGC- $1 \alpha$ 's expression. For example, our results show marked renal ischemia but, unlike in models of ischemia-reperfusion injury, little cell death. The effect of renal ischemia on PGC- $1 \alpha$ regulation has not been fully studied, but may also be important based on data from non-renal models of ischemia (45). Second, experiments are needed to determine whether the early suppression of PGC- $1 \alpha$ in sepsis is adaptive or injurious to tubular epithelium; this question has implications for the timing of potential AKI therapies targeting this pathway. Molecules that activate sirtuins or AMP kinase would be of particular interest given prior demonstrations of their direct inductive interactions with PGC-1 $\alpha(46,47)$. Finally, the fate of damaged tubular mitochondria should also be investigated - for example, induction of mitophagy may be important to prepare the injured tubular cell for recovery.

In summary, the current work provides a molecular and physiological framework for understanding how acute renal impairment in sepsis can arise in the absence of cell death. Restored expression of the mitochondrial biogenesis factor PGC- $1 \alpha$ appears to be necessary for recovery from endotoxemic AKI, suggesting a novel pathway for future therapeutic studies.

\section{Methods}

\section{Mouse studies}

Mice. Eight-week-old male C57BL/6J mice were purchased from The Jackson Laboratory. Global knockouts and mice with the floxed PGC-1 $\alpha$ allele 
have been previously described (21). To generate conditional transgenics targeting deletion of PGC-1 $\alpha$ in the kidney, we bred Sglt2-Cre mice expressing Cre recombinase specifically in the proximal tubule (gift of I. Rubera, University of Nice, Nice, France) with mice with fully floxed $P G C-1 \alpha$. Genotyping of the mice was done as previously described $(21,48)$.

LPS model. Eight- to 10-week-old male mice were used in all experiments. Mice were administered $10 \mathrm{mg} / \mathrm{kg}$ body weight LPS from E. coli serotype O111:B4 (Sigma-Aldrich) i.p. and sacrificed at 18 hours. To study recovery from endotoxemic AKI, we gave subset of mice an i.p. injection of $10 \mathrm{ml} /$ $\mathrm{kg}$ warmed sterile $0.9 \%$ saline 18 hours after LPS, then sacrificed them at 42 hours after the initial LPS injection.

CLP model. All CLP experiments were performed by a single operator. Abdomens of 10- to 12-week-old male mice were prepped in sterile fashion. Using 1\%-3\% inhaled isoflurane anesthesia, we performed a 1-cm midline laparotomy. After mobilization of the cecum, its base was ligated, and a single 21-gauge needle puncture was placed at its distal tip. Stool content $(1 \mathrm{~mm})$ was gently expressed through the perforation. Bowel contents were then replaced, abdomen was closed with two layers, and $1 \mathrm{ml}$ of warm sterile saline was given subcutaneously to resuscitate mice from the procedure. At 18 hours after the procedure, mice were given an additional $10 \mathrm{ml} / \mathrm{kg}$ of sterile saline subcutaneously. They were sacrificed for tissue collection at the indicated times.

\section{Renal function measurements}

Whole blood samples were allowed to clot overnight at room temperature before being centrifuged at $700 \mathrm{~g}$ for 10 minutes and collection of the supernatant serum. Serum BUN and creatinine measurements were obtained using the i-STAT Portable Clinical Analyzer and CHEM8+ cartridges (Abbott Laboratories).

\section{BOLD MRI}

BOLD MRI capitalizes on the difference in magnetic spins between oxyhemoglobin and deoxyhemoglobin using a gradient-echo sequence at different echo times to determine the spin-spin relaxation rate $(\mathrm{R} 2 *=1 / \mathrm{T} 2 *$, such that the derived parameter, $\mathrm{R} 2$ *, is directly proportional to the local deoxyhemoglobin content (49). BOLD MRI of the kidney has been shown to be a sensitive technique for following regional changes in tissue oxygenation in humans and rodents $(15,50)$. These studies were performed at 4.7 Tesla (BioSpec 47/40; Bruker Biospin) using a transmit/receive birdcage coil (inner diameter, $36 \mathrm{~mm}$ ). Animals were anesthetized with $1 \%-2 \%$ isoflurane via a nose cone and placed in prone position, headfirst, with the kidneys centered with respect to the center of the coil. Axial T2*-weighted images were acquired using a multi-gradient echo (MGE) pulse sequence with repetition time $(\mathrm{TR})=400 \mathrm{~ms}$, echo time $(\mathrm{TE})=4-42.5 \mathrm{~ms}$, 8 echo images, matrix size $=256 \times 256$, field of view $(\mathrm{FOV})=3.072 \times 3.072 \mathrm{~cm}$, number of excitations $(\mathrm{NEX})=8$, and slice thickness $=0.5 \mathrm{~mm}$. MRI Mapper software (MIT and Beth Israel Deaconess Medical Center, Boston, Massachusetts, USA) was used to generate $\mathrm{R} 2 *$ maps from $\mathrm{T} 2$ *-weighted images. A region of interest (ROI) was drawn over the outer boundary of renal cortex in the central section of the kidney in the axial plane, and R2* mean and standard deviation were obtained for all pixels included in the ROI. In a separate experiment, the left renal artery of a normal mouse was ligated and imaged using the BOLD technique to demonstrate the effect of hypoxia.

\section{Micro-ultrasound}

Mice were lightly anesthetized with $0.5 \%-1.0 \%$ inhaled isoflurane in medical air and studied by a single operator blinded to the experimental protocol. Following induction, mice were placed on a heated stage and monitored closely for core temperature as well as ECG and respiration. Anesthesia parameters were adjusted accordingly. High-frequency, high- resolution digital imaging platform with linear array technology was used for the study (Vevo 2100, Visual Sonics), equipped with high-frequency linear array probe MS550D (22-55 MHz). Images of anatomical structures as well as Doppler tracings were digitally recorded and analyzed using the manufacturer's software package. For each study, a software-generated average ( $n=3$ cardiac cycles) velocity-time integral was multiplied by the cross-sectional area (modeled as a circle with measured diameter) and ECG-derived heart rate to calculate renal artery blood flow as $\mathrm{mm}^{3} / \mathrm{min}$, which was then converted to $\mathrm{ml} / \mathrm{min}$.

\section{In situ enzyme chemistry}

After removal, kidneys were bi-valved and frozen immediately in isopentane cooled in liquid nitrogen. The tissues were cryosectioned ( $6 \mu \mathrm{m}$ thick) and stained for NADH and COX activities, as described previously (51).

\section{Histology and electron microscopy}

Kidneys were perfusion fixed with $1.25 \%$ glutaraldehyde in $0.1 \mathrm{M}$ phosphate buffer ( $\mathrm{pH}$ 7.4). Kidneys were cut in both sagittal and horizontal cross sections for image analysis. The tissues were treated as described previously (52). Briefly, $1-\mu \mathrm{m}$ sections were dried overnight at $45^{\circ} \mathrm{C}$ on gelatin-coated slides. Slides were then stained at $60^{\circ} \mathrm{C}$ for 2 hours in Giemsa, cooled to room temperature, dehydrated, cleaned in xylene, and mounted in Permount. We used a JEOL 1011 Transmission Electron Microscope, with a Hamamatsu Orca-HR Digital Camera and Advanced Microscopy Techniques (AMT) Corp. image capture system.

\section{In situ hybridization}

The probes for PGC-1 $\alpha$ were obtained by subcloning a 661-bp region of the mouse PGC-1 $\alpha$ cDNA (nucleotides 646-1,307) into pBluescript KS+ vector (Stratagene) and transcribed in vitro to produce antisense and sense riboprobes labeled with digoxigenin. In situ hybridization on cryosections was performed as described previously (53).

\section{Gene expression profiling and analyses}

For transcriptional profiling, total RNA was hybridized to Affymetrix HT Mouse Genome 430 arrays using protocols and quality controls previously described (54). Scanned array images were normalized using the robust multichip average algorithm in Bioconductor. Present/absent/marginal calls were obtained using MAS5 (http://www.bioconductor.org). Before PCA, data were filtered by removing all probes that were absent in all groups. The unsupervised analysis was performed using PCA, which projects multivariate data objects onto a lower dimensional space while retaining as much of the variance as possible.

To identify differentially expressed genes across the 4 conditions (baseline, early injury, persistent injury, and recovery), a 1-way ANOVA model was employed in Bioconductor with a threshold of $P<0.001$ for statistical significance. This list of significantly differentially expressed genes was further filtered to retain genes with more than 5 standard deviations of variance across groups to reduce the false-positive rate. To identify gene expression profiles that were functionally related to the biological state of interest and coordinated across the 4 conditions, we then applied SOM clustering on transcript expression values to 50 target groups. SOM allows the grouping of gene expression patterns into an imposed structure in which adjacent clusters are related, thereby identifying sets of genes that follow certain expression patterns across different conditions (55).

IPA 7.0 (Ingenuity Systems) was then applied to the strongest SOM patterns to identify biological pathways significantly overrepresented in SOM clusters. The knowledge base of the Ingenuity software consists of functions, pathways, and network models derived by systemically cataloguing the peer-reviewed scientific literature (see http://www.ingenuity.com for 
more details). It calculates a $P$ value for each pathway according to the fit of user data to the IPA canonical pathways database, indicating the likelihood of finding pathways by random chance.

The microarray data have been deposited in NCBI Gene Expression Omnibus, accession number GSE30576.

\section{Quantitative PCR}

Total RNA was extracted using TRIzol (Invitrogen), followed by clean-up using the RNeasy Mini Kit with on-column DNase digestion (QIAGEN) per the manufacturer's instructions. Total RNA was then reverse transcribed to CDNA, and PCR reactions were performed in duplicate using the ABI 7500 Fast Real-Time PCR System (Applied Biosystems). Relative expression levels were determined using the comparative threshold method.

\section{Western blot analysis}

Kidney lysates were prepared by homogenization in ice-cold RIPA buffer (Boston BioProducts) supplemented with protease inhibitors (Roche Diagnostics), $1 \mathrm{mM}$ EDTA, $1 \mathrm{mM} \mathrm{Na}_{3} \mathrm{VO}_{4}$, and $1 \mathrm{mM} \mathrm{NaF}$. Lysates were sonicated and centrifuged at $8,000 \mathrm{~g}$ for 10 minutes at $4{ }^{\circ} \mathrm{C}$, and supernatants were collected. Electrophoresis, transfer, immunoblotting, detection, and image acquisition were performed as previously described (56). Anti-PGC-1 $\alpha$ (Cayman Chemicals) or anti-cytochrome $c$ oxidase (Cell Signaling Technology) antibodies were used.

\section{Oxygen consumption experiments}

Passage 2-6 primary human RPTECs (Lonza) were seeded in an XF 24-well cell culture plate (Seahorse Bioscience) at $2 \times 10^{4}$ cells per well and grown in regular medium, treated with TNF- $\alpha$ at the indicated concentrations, and studied in unbuffered DMEM supplemented with $7 \mathrm{mM}$ glucose and $10 \mathrm{mM}$ pyruvate. Oxygen consumption rates $(\mathrm{pmol} / \mathrm{min}$ ) were assessed using an XF Flux Analyzer (Seahorse Bioscience) at baseline, after the addition of the ATP synthase inhibitor oligomycin $(10 \mu \mathrm{M})$, after the addition of the uncoupling agent 2,4-dinitrophenol $(150 \mu \mathrm{M})$, and again after addition of the complex I inhibitor rotenone $(2 \mu \mathrm{M})$. These pharmacological manip- ulations enabled us to isolate the effects on mitochondrial oxygen consumption from other cellular sources of oxygen utilization. For adenovirus experiments, a GFP control backbone virus or adenovirus encoding murine PGC- $1 \alpha$ was applied to RPTECs 48 hours prior to an experiment at 5- to 10 -fold multiplicity of infection. Efficiency was confirmed by GFP visualization and transgene expression by quantitative PCR.

\section{Statistics}

Data were analyzed by unpaired 2-sided $t$ test, Mann-Whitney $U$ test, or ANOVA for multiple comparisons. Correlations were performed with the Spearman method. Results are presented as mean \pm SEM using GraphPad Prism. A $P$ value of less than 0.05 was considered significant.

\section{Study approval}

All animal experiments were performed according to procedures approved by Beth Israel Deaconess Medical Center's Institutional Animal Care and Use Committee.

\section{Acknowledgments}

This work was supported by the American Society of Nephrology's Gottschalk Award, the Klarman Family Scholarship, and NIH grants DK069316 and HL093234 to S.M. Parikh.

Received for publication April 22, 2011, and accepted in revised form July 13, 2011.

Address correspondence to: Samir M. Parikh, Beth Israel Deaconess Medical Center, 330 Brookline Avenue, RN280C, Boston, Massachusetts 02215, USA. Phone: 617.667.5813; Fax: 617.667.2646; E-mail: sparikh1@bidmc.harvard.edu.

Portions of the data were presented in abstract form at the American Society of Nephrology annual meeting in Denver, Colorado, USA, November 16-21, 2010.
1. Hoste EA, Kellum JA. Acute kidney injury: epidemiology and diagnostic criteria. Curr Opin Crit Care. 2006;12(6):531-537.

2. Martin GS, Mannino DM, Eaton S, Moss M. The epidemiology of sepsis in the United States from 1979 through 2000. N Engl J Med. 2003; 348(16):1546-1554.

3. Schrier RW, Wang W. Acute renal failure and sepsis. NEngl J Med. 2004;351(2):159-169.

4. Bellomo R, et al. Intensity of continuous renalreplacement therapy in critically ill patients. $N$ Engl JMed. 2009;361(17):1627-1638.

5. Palevsky PM, et al. Intensity of renal support in critically ill patients with acute kidney injury. NEngl J Med. 2008;359(1):7-20.

6. Doi K, Leelahavanichkul A, Yuen PS, Star RA. Animal models of sepsis and sepsis-induced kidney injury. J Clin Invest. 2009;119(10):2868-2878.

7. Langenberg C, Bagshaw SM, May CN, Bellomo R. The histopathology of septic acute kidney injury: a systematic review. Crit Care. 2008;12(2):R38.

8. Hotchkiss RS, Karl IE. The pathophysiology and treatment of sepsis. NEngl J Med. 2003;348(2):138-150.

9. Cumming AD, Driedger AA, McDonald JW, Lindsay RM, Solez K, Linton AL. Vasoactive hormones in the renal response to systemic sepsis. Am J Kidney Dis. 1988;11(1):23-32.

10. Miyaji T, et al. Ethyl pyruvate decreases sepsisinduced acute renal failure and multiple organ damage in aged mice. Kidney Int. 2003;64(5):1620-1631.

11. Sjostrand F, Rhodin J. The ultrastructure of the proximal convoluted tubules of the mouse kidney as revealed by high resolution electron microscopy.
Exp Cell Res. 1953;4(2):426-456.

12. Weidemann MJ, Krebs HA. The fuel of respiration of rat kidney cortex. Biochem J. 1969;112(2):149-166.

13. Thadhani R, Pascual M, Bonventre JV. Acute renal failure. NEngl J Med. 1996;334(22):1448-1460.

14. Puigserver P, Wu Z, Park CW, Graves R, Wright M, Spiegelman BM. A cold-inducible coactivator of nuclear receptors linked to adaptive thermogenesis. Cell. 1998;92(6):829-839.

15. Prasad PV, Edelman RR, Epstein FH. Noninvasive evaluation of intrarenal oxygenation with BOLD MRI. Circulation. 1996;94(12):3271-3275.

16. Rosenberger $C$, et al. Expression of hypoxia-inducible factor- $1 \alpha$ and $-2 \alpha$ in hypoxic and ischemic rat kidneys. J Am Soc Nephrol. 2002;13(7):1721-1732.

17. Feingold KR, Wang Y, Moser A, Shigenaga JK Grunfeld C. LPS decreases fatty acid oxidation and nuclear hormone receptors in the kidney. J Lipid Res. 2008;49(10):2179-2187.

18. Maitra U, Chang S, Singh N, Li L. Molecular mechanism underlying the suppression of lipid oxidation during endotoxemia. Mol Immunol. 2009; 47(2-3):420-425.

19. Wang Y, Moser AH, Shigenaga JK, Grunfeld C, Feingold KR. Downregulation of liver $X$ receptor- $\alpha$ in mouse kidney and HK-2 proximal tubular cells by LPS and cytokines. J Lipid Res. 2005;46(11):2377-2387.

20. Wu Z, et al. Mechanisms controlling mitochondrial biogenesis and respiration through the thermogenic coactivator PGC-1. Cell. 1999;98(1):115-124.

21. Lin J, et al. Defects in adaptive energy metabolism with CNS-linked hyperactivity in PGC-1 $\alpha$ null mice. Cell. 2004;119(1):121-135.
22. Weinberg JM. Mitochondrial biogenesis in kidney disease. JAm Soc Nephrol. 2011;22(3):431-436.

23. Schwartz D, et al. Inhibition of constitutive nitric oxide synthase (NOS) by nitric oxide generated by inducible NOS after lipopolysaccharide administration provokes renal dysfunction in rats. J Clin Invest. 1997;100(2):439-448.

24. Albert M, Losser MR, Hayon D, Faivre V, Payen D. Systemic and renal macro- and microcirculatory responses to arginine vasopressin in endotoxic rabbits. Crit Care Med. 2004;32(9):1891-1898.

25. Cunningham PN, Wang Y, Guo R, He G, Quigg RJ. Role of Toll-like receptor 4 in endotoxininduced acute renal failure. I Immunol. 2004; 172(4):2629-2635.

26. Cunningham PN, Dyanov HM, Park P, Wang J, Newell KA, Quigg RJ. Acute renal failure in endotoxemia is caused by TNF acting directly on TNF receptor-1 in kidney. J Immunol. 2002;168(11):5817-5823.

27. Dear JW, et al. Sepsis-induced organ failure is mediated by different pathways in the kidney and liver: acute renal failure is dependent on MyD88 but not renal cell apoptosis. Kidney Int. 2006;69(5):832-836.

28. Wang W, et al. Role of leptin deficiency in early acute renal failure during endotoxemia in ob/ob mice. J Am Soc Nephrol. 2004;15(3):645-649.

29. Brealey D, et al. Mitochondrial dysfunction in a long-term rodent model of sepsis and organ failure. Am J Physiol Regul Integr Comp Physiol. 2004; 286(3):R491-R497.

30. Brealey D, et al. Association between mitochondrial dysfunction and severity and outcome of septic shock. Lancet. 2002;360(9328):219-223. 
31. Gellerich FN, et al. Mitochondrial dysfunction in sepsis: evidence from bacteraemic baboons and endotoxaemic rabbits. Biosci Rep. 2002;22(1):99-113.

32. d'Avila JC, Santiago AP, Amancio RT, Galina A, Oliveira MF, Bozza FA. Sepsis induces brain mitochondrial dysfunction. Crit Care Med. 2008;36(6):1925-1932.

33. Boekstegers P, Weidenhofer S, Pilz G, Werdan K Peripheral oxygen availability within skeletal muscle in sepsis and septic shock: comparison to limited infection and cardiogenic shock. Infection. 1991; 19(5):317-323.

34. Rosser DM, Stidwill RP, Jacobson D, Singer M. Oxygen tension in the bladder epithelium rises in both high and low cardiac output endotoxemic sepsis. J Appl Physiol. 1995;79(6):1878-1882.

35. Dyson A, Bezemer R, Legrand M, Balestra G, Singer $M$, Ince C. Microvascular and interstitial oxygen tension in the renal cortex and medulla studied in a 4-h rat model of LPS-induced endotoxemia. Shock. 2011;36(1):83-89.

36. Johannes T, Mik EG, Nohe B, Raat NJ, Unertl KE, Ince $\mathrm{C}$. Influence of fluid resuscitation on renal microvascular PO2 in a normotensive rat model of endotoxemia. Crit Care. 2006;10(3):R88.

37. Heemskerk AE, et al. Renal function and oxygen consumption during bacteraemia and endotoxaemia in rats. Nephrol Dial Transplant. 1997;12(8):1586-1594.

38. Haden DW, et al. Mitochondrial biogenesis restores oxidative metabolism during Staphylococcus aureus sepsis. Am J Respir Crit Care Med. 2007 176(8):768-777.

39. Handschin C, Spiegelman BM. Peroxisome proliferator-activated receptor $\gamma$ coactivator 1 coactiva- tors, energy homeostasis, and metabolism. Endocr Rev. 2006;27(7):728-735.

40. St-Pierre J, et al. Suppression of reactive oxygen species and neurodegeneration by the PGC-1 transcriptional coactivators. Cell. 2006;127(2):397-408.

41. Niaudet P, Rotig A. Renal involvement in mitochondrial cytopathies. Pediatr Nephrol. 1996; 10(3):368-373.

42. Li S, et al. Transgenic expression of proximal tubule peroxisome proliferator-activated receptor- $\alpha$ in mice confers protection during acute kidney injury. Kidney Int. 2009;76(10):1049-1062.

43. Brooks C, Wei Q, Cho SG, Dong Z. Regulation of mitochondrial dynamics in acute kidney injury in cell culture and rodent models. J Clin Invest. 2009; 119(5):1275-1285.

44. Rasbach KA, Schnellmann RG. PGC-1 $\alpha$ over-expression promotes recovery from mitochondrial dysfunction and cell injury. Biochem Biophys Res Commun. 2007;355(3):734-739.

45. Arany $Z$, et al. HIF-independent regulation of VEGF and angiogenesis by the transcriptional coactivator PGC-1 $\alpha$. Nature. 2008;451(7181):1008-1012.

46. Jager S, Handschin C, St-Pierre J, Spiegelman BM. AMP-activated protein kinase (AMPK) action in skeletal muscle via direct phosphorylation of PGC- $1 \alpha$. Proc Natl Acad Sci U S A. 2007;104(29):12017-12022.

47. Lagouge $M$, et al. Resveratrol improves mitochondrial function and protects against metabolic disease by activating SIRT1 and PGC-1 $\alpha$. Cell. 2006; 127(6):1109-1122.

48. Rubera I, et al. Specific Cre/Lox recombination in the mouse proximal tubule. J Am Soc Nephrol. 2004;
15(8):2050-2056.

49. Ogawa S, Lee TM, Kay AR, Tank DW. Brain magnetic resonance imaging with contrast dependent on blood oxygenation. Proc Natl Acad Sci US A. 1990; 87(24):9868-9872.

50. Prasad PV, Priatna A, Spokes K, Epstein FH. Changes in intrarenal oxygenation as evaluated by BOLD MRI in a rat kidney model for radiocontrast nephropathy. J Magn Reson Imaging. 2001;13(5):744-747.

51. Lebrecht D, Setzer B, Rohrbach R, Walker UA. Mitochondrial DNA and its respiratory chain products are defective in doxorubicin nephrosis. Nephrol Dial Transplant. 2004;19(2):329-336

52. Heyman SN, Stillman IE, Brezis M, Epstein FH, Spokes K, Rosen S. Chronic amphotericin nephropathy: morphometric, electron microscopic, and functional studies. J Am Soc Nephrol. 1993;4(1):69-80.

53. Braissant O, Wahli W. Differential expression of peroxisome proliferator-activated receptor- $\alpha,-\beta$, and $-\gamma$ during rat embryonic development. Endocrinology. 1998;139(6):2748-2754.

54 . Horst D, et al. Requirement of the epitheliumspecific Ets transcription factor Spdef for mucous gland cell function in the gastric antrum.J Biol Chem. 2010;285(45):35047-35055.

55. Tamayo $\mathrm{P}$, et al. Interpreting patterns of gene expression with self-organizing maps: methods and application to hematopoietic differentiation. Proc Natl Acad Sci U S A. 1999;96(6):2907-2912.

56. David S, et al. Effects of a synthetic PEG-ylated Tie-2 agonist peptide on endotoxemic lung injury and mortality. Am J Physiol Lung Cell Mol Physiol. 2011; 300(6):L851-L862. 